\title{
Aplicação da Técnica Total Variation Denoising (TVD) na Regularização de um Problema Inverso de Estimativa de Fluxo de Calor
}

\section{Application of the Total Variation Denoising (TVD) Technique to the Regularization of a Heat Flux Estimation Inverse Problem}

Eiji Watanabe1, Gabriel Teixeira Soares das Neves², Luiz Alberto da Silva Abreu³, Diego Campos Knupp ${ }^{4}$, Antônio José da Silva Neto ${ }^{5}$

\section{RESUMO}

Este trabalho aborda o problema de estimativa de fluxo de calor com variação espacial unidimensional e constante no tempo aplicado a uma das superfícies de uma placa de aço inoxidável. O problema direto é resolvido empregando o método de diferenças finitas. A solução do problema inverso é obtida com o emprego de uma abordagem Bayesiana, utilizando o Método de Monte Carlo com Cadeias de Markov. Para a regularização do problema é proposta uma aplicação da técnica Total Variation Denoising (TVD). A abordagem utilizada foi capaz de estimar, satisfatoriamente, o fluxo de calor aplicado, demonstrando a robustez desta técnica de regularização na solução deste problema inverso.

Palavras-chave: Problemas Inversos, Estimativa de Fluxo de Calor, Inferência Bayesiana, Total Variation Denoising (TVD)

\section{ABSTRACT}

This work addresses the inverse problem of estimating the spacewise varying heat flux applied to the surface of a thin plate. The direct problem is solved with the finite difference method. The inverse problem is formulated using a Bayesian approach, and the solution is obtained with a Markov Chain Monte Carlo method. In order to regularize the noisy experimental data, the Total Variation Denoising (TVD) technique is proposed. The approach here proposed yielded satisfactory results, demonstrating the robustness of the TVD technique in regularizing this inverse problem solution.

Keywords: Inverse Problems, Heat Flux Estimation, Bayesian Inference, Total Variation Denoising (TVD)
${ }^{1}$ Graduando em Engenharia Mecânica - Instituto

Politécnico/Universidade do Estado do Rio de Janeiro.

E-mail: eijiwwf@gmail.com

${ }^{2}$ MSc. - Instituto Federal de Educação Ciência e Tecnologia Fluminense, Doutorando em Modelagem Computacional Instituto

Politécnico/Universidade do Estado do Rio de Janeiro.

${ }^{3}$ DSc. - Instituto

Politécnico/Universidade do Estado do Rio de Janeiro.

${ }^{4}$ DSc. - Instituto

Politécnico/Universidade do Estado do Rio de Janeiro.

${ }^{5}$ PhD. - Instituto

Politécnico/Universidade do Estado do Rio de Janeiro. 


\section{INTRODUÇÁAO}

Com o avanço da tecnologia e, como consequência, com o fenômeno da miniaturização, atendendo a demanda de componentes eletrônicos cada vez menores, como, por exemplo, microchips e nanochips, surgem questões diversas na logística envolvidas na produção dessas peças. Problemas de superaquecimento e, consequentemente, dissipação de calor são obstáculos enfrentados constantemente nas indústrias. Dessa forma, soluções no sentido de identificar com mais precisão e agilidade possíveis fluxos de calor em componentes eletrônicos de tamanho reduzido assumem papel de destaque tanto no ambiente acadêmico quanto no industrial.

A estimativa de fontes térmicas e fluxos de calor a partir de medições de campos de temperaturas é caracterizada como um problema inverso. Problemas desse tipo são estudados há décadas através de técnicas determinísticas como o método de regularização iterada de Alifanov (SILVA NETO, ÖZISIK, 1993; SU, SILVA NETO, 2001). Nos últimos anos, a popularização de abordagens Bayesianas, como o Método de Monte Carlo com Cadeias de Markov (MCMC), permitiu o estudo de novos problemas (MESCOLIN et al., 2016; ABREU, ORLANDE, COLAÇO, 2016).

Em problemas inversos envolvendo a estimativa de funções, o custo computacional pode ser inviável para determinadas situações e as soluções obtidas podem se mostrar pouco regularizadas. A partir de abordagens Bayesianas, encontrar métodos adaptativos e informações a priori que ajudem a contornar esses problemas tem sido assunto central em diversos trabalhos no estudo de problemas inversos. Diversas abordagens, com diferentes aspectos, foram apresentadas no decorrer dos anos, como prioris Gaussianas e prioris do tipo Markov Random Fields (KAIPIO, SOMERSALO, 2004).

O presente trabalho aborda o caso do problema de estimativa de fluxo de calor unidimensional e constante no tempo aplicado a uma placa de aço inoxidável retangular. É proposta a utilização da técnica Total Variation Denoising (TVD) para regularização do problema.

\section{METODOLOGIA}

\subsection{PROBLEMA DIRETO}

Considere o problema de condução de calor transiente unidimensional em uma placa termicamente fina, de dimensões $L_{x} \times L_{y} \times L_{z}$ sujeita à condição inicial $T(x, y, z, 0)=T_{\infty}$. 
Um fluxo de calor que varia na direção $x, q(x)$, é aplicado na superfície inferior $(z=0)$, enquanto a superfície superior $\left(z=L_{z}\right)$ é sujeita à troca de calor por convecção com o ambiente a uma temperatura $T_{\infty}$ e coeficiente de troca térmica $h$, como apresentado na Figura 1. É assumido que a espessura, $L_{z}$, da placa é muito menor do que sua largura, $L_{y}$, e seu comprimento, $L_{x}$, e, portanto, as perdas de calor nas extremidades são desprezadas (ORLANDE et al., 2014).

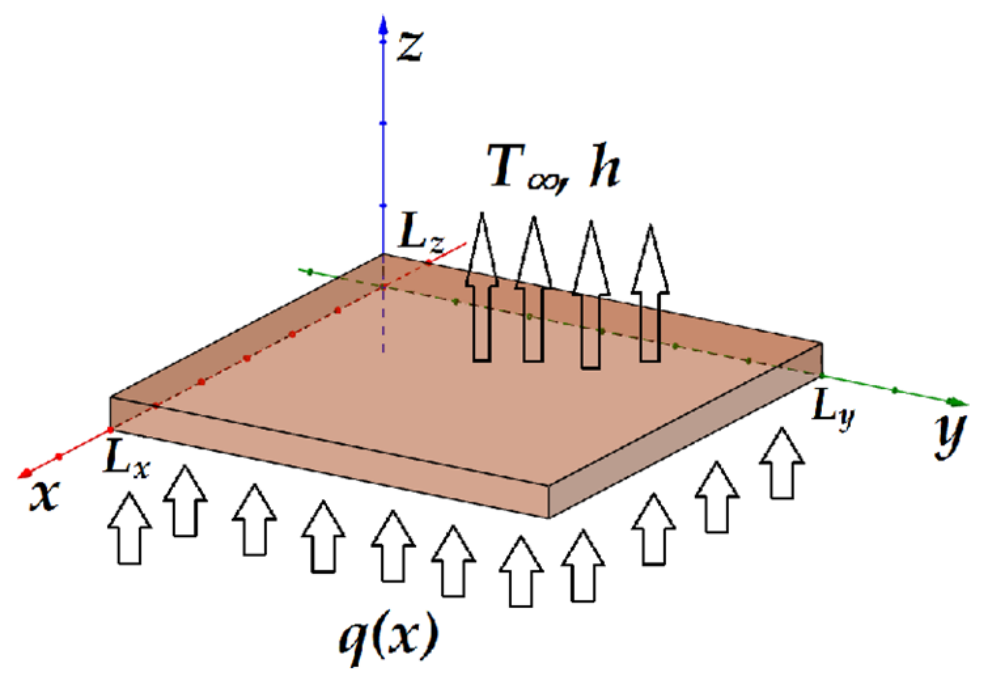

Figura 1. - Representação do problema físico

Considerando que não exista geração de calor volumétrico no interior da placa, a equação para a condução de calor no regime transiente é dada pela Equação 1 (BERGMAN et al., 2014):

$$
\frac{\partial}{\partial x}\left(k \frac{\partial T}{\partial x}\right)+\frac{\partial}{\partial y}\left(k \frac{\partial T}{\partial y}\right)+\frac{\partial}{\partial z}\left(k \frac{\partial T}{\partial z}\right)=\rho c_{p} \frac{\partial T(x, y, z, t)}{\partial t}
$$

onde $\rho$ é a massa específica $\left(k g / \mathrm{m}^{3}\right), c_{p}$ é o calor específico $(\mathrm{J} /(\mathrm{kg} \cdot K))$, e $k$, a condutividade térmica $(W /(m \cdot K))$.

As condições de contorno e inicial impostas ao problema são dadas por

$$
-\left.k \frac{\partial T}{\partial z}\right|_{z=0}=q(x)
$$




$$
\begin{gathered}
-\left.k \frac{\partial T}{\partial z}\right|_{z=L_{z}}=h\left(T-T_{\infty}\right) \\
-\left.k \frac{\partial T}{\partial x}\right|_{x=0}=-\left.k \frac{\partial T}{\partial y}\right|_{y=0}=0 \\
-\left.k \frac{\partial T}{\partial x}\right|_{x=L_{x}}=-\left.k \frac{\partial T}{\partial y}\right|_{y=L_{y}}=0
\end{gathered}
$$

e

$$
T(x, y, z, 0)=T_{\infty}
$$

onde $L_{x}, L_{y}$ e $L_{z}$ são, respectivamente, a largura da placa em $x$ e $y$, e a espessura em $z$, e $T_{\infty}$ representa a temperatura ambiente.

O fluxo de calor imposto na superfície $z=0$ é escrito como

$$
q(x)= \begin{cases}500 \mathrm{~W} / \mathrm{m}^{2} & \text { se } 0,02 \mathrm{~m} \leq x \leq 0,06 \mathrm{~m} \\ 0 \mathrm{~W} / \mathrm{m}^{2} & \text { se } x<0,02 \mathrm{~m} \text { ou } x>0,06 \mathrm{~m}\end{cases}
$$

Considerando que o fluxo é constante através da direção y do domínio, o termo difusivo nesta direção pode ser desprezado. Sendo assim

$$
\frac{\partial T}{\partial y}=0
$$

o que é compatível com as condições de contorno impostas em $y=0$ e $y=L_{y}$.

Na direção $z$ será aplicado o método da capacitância global (Lumped) com a condição de que o número de Biot seja menor do que 0,1. A fim de validar o método, esse número é calculado com a condutividade térmica $k=15,1 \mathrm{~W} / \mathrm{mK}$, espessura da placa $L_{z}=0,002 \mathrm{~m}$ e coeficiente de troca térmica por convecção $h=15 \mathrm{~W} / \mathrm{m}^{2} \mathrm{~K}$. 


$$
B i=\frac{h L_{z}}{k}=\frac{15 \cdot 0,002}{15,1} \approx 0,002
$$

Como $B i<0,1$, o método é válido e pode ser usado. Definindo

$$
\bar{T}(x, y, z, t)=\frac{1}{L_{z}} \int_{0}^{L_{z}} T(x, y, z, t) d z
$$

em que $\bar{T}$ é a temperatura média na direção $z$, aplica-se o operador $\frac{1}{L_{z}} \int_{0}^{L_{z}}(\cdot) d z$ na Equação 1, nas condições de contorno e na condição inicial, obtendo-se

$$
\frac{\partial}{\partial x}\left(k \frac{\partial \bar{T}}{\partial x}\right)+\frac{1}{L_{z}}\left(\left.k \frac{\partial \bar{T}}{\partial z}\right|_{z=L_{z}}-\left.k \frac{\partial \bar{T}}{\partial z}\right|_{z=0}\right)=\rho c_{p} \frac{\partial \bar{T}(x, t)}{\partial t}
$$

ou ainda, considerando a condutividade térmica constante,

$$
k \frac{\partial^{2} \bar{T}}{\partial x^{2}}+\frac{1}{L_{z}}\left(-h\left(\bar{T}-T_{\infty}\right)+q(x)\right)=\rho c_{p} \frac{\partial \bar{T}(x, t)}{\partial t}
$$

O problema proposto foi resolvido numericamente aplicando o Método de Diferenças Finitas por meio do software Wolfram Mathematica®. Utilizando aproximações de segunda ordem no espaço e de primeira ordem no tempo, a Equação 12 é aproximada explicitamente por

$$
T_{i}^{n+1}=\varphi\left(T_{i-1}^{n}+T_{i+1}^{n}\right)+(1-2 \varphi) T_{i}^{n}+\frac{\varphi \Delta x^{2}}{k L_{z}}\left(-h\left(T_{i}^{n}-T_{\infty}\right)+q(x)\right)
$$

Por outro lado, sua fórmula implícita é escrita como 


$$
-\varphi T_{i-1}^{n+1}+\left(2 \varphi+\frac{\varphi \Delta x^{2}}{k L_{z}} h+1\right) T_{i}^{n+1}-\varphi T_{i+1}^{n+1}=\frac{\varphi \Delta x^{2}}{k L_{z}}\left(h T_{\infty}+q(x)\right)+T_{i}^{n}
$$

$\operatorname{com} \varphi=\frac{k \Delta t}{\rho c_{p} \Delta x^{2}}$

A convergência da solução do método foi verificada com a solução gerada intrinsecamente pelo MatLab® através do PDE Toolbox.

\subsection{PROBLEMA INVERSO}

Para a formulação do problema inverso são utilizados dados experimentais simulados, adicionando ruído à solução do problema direto, fornecendo um total de $\mathrm{D}=\mathrm{N}_{\mathrm{x}} \times N_{t}$ medidas, sendo $N_{x}$ sensores e $N_{t}$ medidas obtidas com cada sensor. $O$ vetor de dados experimentais pode ser expresso como

$$
Y^{T}=\left(Y_{1}, Y_{2}, \ldots, Y_{D}\right)
$$

onde $Y_{i}, i=1,2, \ldots, D$, corresponde à medição em uma posição da malha em um instante de tempo.

O fluxo de calor $q(x)$ é estimado sobre uma malha com $N P_{x}$ nós, levando a $N P_{x}$ parâmetros a serem estimados, $q_{i}, i=1,2, \ldots, N P_{x}$. O vetor de estimativas para os parâmetros procurados pode ser escrito como

$$
P^{T}=\left(q_{1}, q_{2}, \ldots, q_{N P_{x}}\right)
$$

Para a solução do problema inverso foi utilizada uma abordagem Bayesiana, pois possibilita combinar informações a priori no cálculo das estimativas através do teorema de Bayes (KAIPIO, SOMERSALO, 2004), característica importante para a regularização de problemas em que muitos parâmetros são estimados. A solução do problema consiste numa curva de densidade de probabilidade a posteriori, expressa por

$$
\pi_{\text {post }}(P)=\pi(P \mid Y)=\frac{\pi_{\text {prior }}(P) \pi(Y \mid P)}{\pi(Y)}
$$


onde $\pi_{\text {post }}(P)$ é a distribuição posterior de probabilidade dos parâmetros $P, \pi_{\text {prior }}(P)$ é a distribuição de probabilidades dos dados a priori, $\pi(Y)$ a distribuição marginal de probabilidade dos dados experimentais, que desempenha papel de constante de normalização, e $\pi(Y \mid P)$, a função de verossimilhança, expressa analiticamente na forma

$$
\pi(Y \mid P)=(2 \pi)^{-D / 2}|W|^{-1 / 2} \times \exp \left[-\frac{1}{2}[Y-T(P)]^{T} W^{-1}[Y-T(P)]\right]
$$

em que $D$ é a quantidade de medidas, $W$ é a matriz de covariâncias dos erros das medidas e $T(P)$ é o vetor contendo a solução do problema direto dados os valores de $P$, nas mesmas posições em que são obtidas as medidas experimentais.

Neste trabalho é utilizado o algoritmo Metropolis-Hastings, descrito detalhadamente por (KAIPIO \& SOMERSALO, 2004), para gerar candidatos para a distribuição a posteriori. A implementação se inicia com a seleção de uma densidade de probabilidade de movimentação $p\left(P^{*}, P^{t-1}\right)$, que é utilizada para gerar um candidato $P^{*}$ para o novo estado da cadeia, dado o estado anterior $P^{t-1}$. Criando assim a sequência $\left\{P^{1}, P^{2}, \ldots, P^{n}\right\}$, que deve convergir para a solução procurada.

A regularização é uma etapa importante na solução de problemas inversos, especialmente quando há muitos parâmetros a serem estimados. Para tanto, é proposta a aplicação da técnica Total Variation Denoising (TVD), inicialmente proposta por (RUDIN et al., 1992), através do algoritmo proposto por (CONDAT, 2013). A técnica consiste num problema de minimização e possui a característica de mostrar eficiência na reconstrução de imagens com gradiente absoluto elevado. Essa característica é similar ao caso de hotspots, onde há fluxos pontuais e de elevada intensidade de calor no domínio. Desse modo, aplicamos o filtro não apenas à imagem de dados experimentais, no intuito de reduzir seus ruídos, mas também aos candidatos gerados dentro do algoritmo Metropolis-Hastings.

O problema de atenuar o ruído de um sinal $y=\left(y_{1}, \ldots, y_{n}\right) \in \mathbb{R}^{n}$, com $n>1$ através do Total Variation Denoising, consiste em calcular o sinal $x \in \mathbb{R}^{n}$ que seja a solução do problema de minimização

$$
\min _{x \in \mathbb{R}^{n}} J(x)=\min _{x \in \mathbb{R}^{n}} \frac{1}{2} \sum_{k=1}^{n}\left|y_{k}-x_{k}\right|^{2}+\lambda \sum_{k=1}^{n-1}\left|x_{k+1}-x_{k}\right|
$$


sendo $\lambda$ o parâmetro de regularização a ser determinado empiricamente. $O$ funcional a ser minimizado, $J(x)$, é fortemente convexo e, portanto, existe solução $x$ e ela é única, para quaisquer dados de entrada $y$ por (CONDAT, 2013).

\section{RESULTADOS}

Foi considerado o caso de uma placa retangular, com dimensões $0,08 m \times 0,04 m$ e $0,002 m$ de espessura, com fluxo de calor imposto em uma região com a área de $0,04 m \times$ $0,04 m$ posicionada geometricamente no centro da placa. A simulação teve duração de 1.200 segundos e as propriedades do material simulado são próximas àquelas dos utilizados na manufatura de chips eletrônicos, com $k=15,1 \mathrm{~W} / \mathrm{mK}$ e $\rho c_{p}=3.866 .400 \mathrm{~J} /$ $m^{3} K$. O coeficiente de transferência de calor escolhido foi de $h=15 \mathrm{~W} / \mathrm{m}^{2} \mathrm{~K}$, com $T_{\infty}=$ $23^{\circ} \mathrm{C}$. O fluxo, em $\mathrm{W} / \mathrm{m}^{2}$, que se pretende estimar é o descrito pela Equação 7, que consiste em uma função com variações abruptas no espaço.

A malha utilizada para estimação do fluxo possui 50 nós, o que leva a 50 parâmetros a serem estimados, $q_{i}$, com $i=1,2, \ldots, 50$, i.e., $N P_{x}=50$ na Equação 16, e os dados experimentais foram simulados adicionando um ruído com distribuição Gaussiana, média zero e desvio padrão $\sigma=0,05^{\circ} \mathrm{C}$ à solução convergida do problema direto calculada via diferenças finitas. As propostas para as estimativas do fluxo de calor a serem utilizadas no MCMC são distribuições normais com desvio padrão $\delta=5 \mathrm{~W} / \mathrm{m}^{2}$. Foram calculados 50.000 estados na cadeia de Markov, sendo os 10.000 primeiros descartados, período conhecido como burn in.

Devido ao elevado número de parâmetros a serem determinados, faz-se necessário o uso de técnicas de regularização para o problema, na forma de informação a priori. Os resultados aqui apresentados comparam três diferentes cenários. O Caso 1 utiliza a Total Variation Denoising apenas como filtro para os dados experimentais; o Caso 2 utiliza a mesma técnica como priori nos candidatos gerados no MCMC; e o Caso 3 combina a utilização do filtro nos dados experimentais e nos candidatos em comparação com o valor exato na direção $x$. 


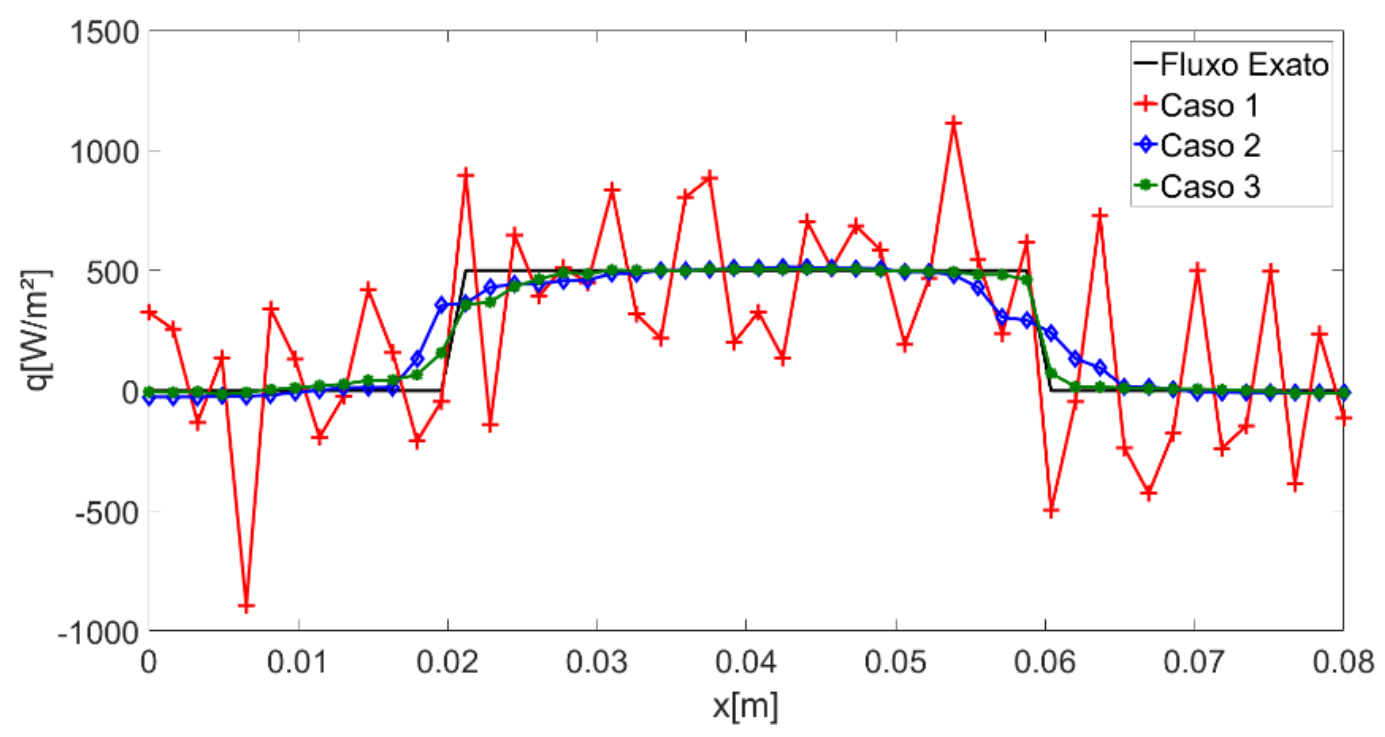

Figura 2. Estimativa do fluxo de calor com os métodos propostos

É possível notar que a aplicação da técnica apenas como filtro nos dados experimentais não é capaz de regularizar a solução do problema inverso. Por outro lado, a utilização da mesma técnica, para $\lambda=0,9$, vide Equação 19, nos candidatos do MCMC, conseguiu produzir uma solução que se aproxima satisfatoriamente da solução exata, sendo que houve certa dificuldade do método em capturar as altas variações do fluxo no espaço. Em compensação, utilizando valores de $\lambda$ iguais a 0,01 e 0,9 no TVD aplicado às medidas e aos candidatos do MCMC, respectivamente, é possível observar um ajuste melhor às altas variações do fluxo.

As Figuras 3(a) e 3(b) mostram a evolução das cadeias de Markov para posições na malha onde se espera estimar valores por volta de $q(x)=0 \mathrm{~W} / \mathrm{m}^{2}$ e $q(x)=500 \mathrm{~W} / \mathrm{m}^{2}$, respectivamente. É possível notar, conforme as cadeias evoluem, suas convergências para valores próximos a $0 \mathrm{~W} / \mathrm{m}^{2}$ e $500 \mathrm{~W} / \mathrm{m}^{2}$, respectivamente. As Figuras 3(c) e 3(d) apresentam os histogramas para os 40.000 últimos estágios das cadeias, para as mesmas posições na malha utilizadas nas figuras anteriores, ficando então centrados em valores ao redor de $-10 \mathrm{~W} / \mathrm{m}^{2}$ e $505 \mathrm{~W} / \mathrm{m}^{2}$, valores próximos aos que se deseja estimar.
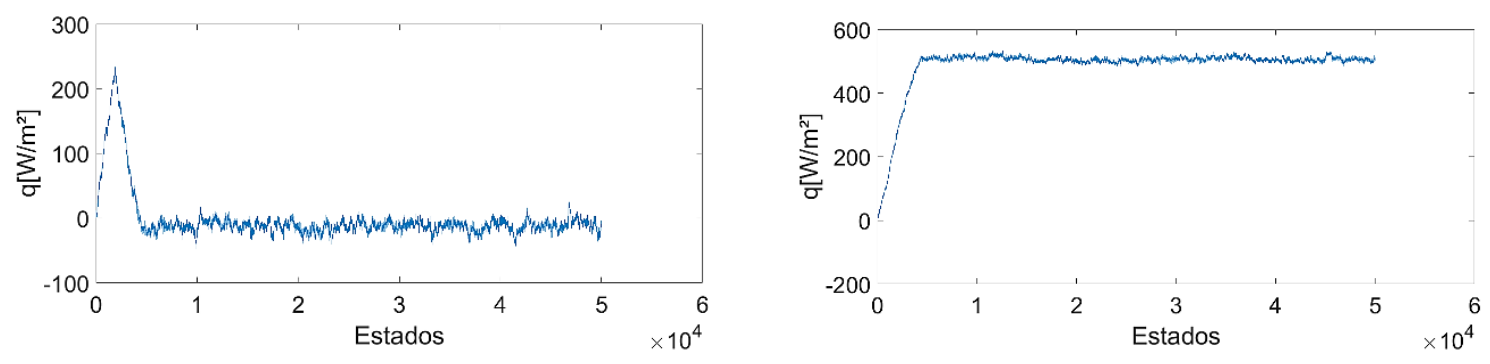
(a)

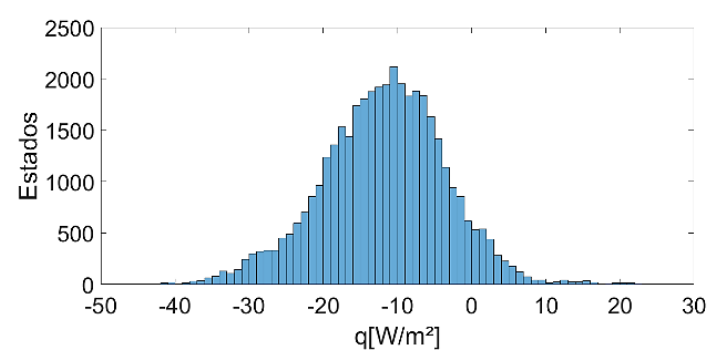

(c) (b)

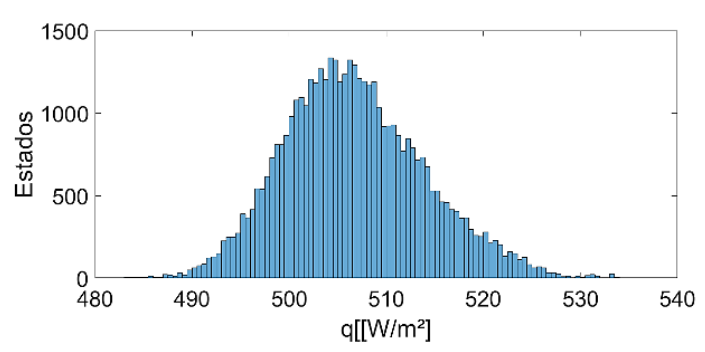

(d)

Figura 3. Evolução das Cadeias de Markov para fluxo $\boldsymbol{q}(\boldsymbol{x})=\mathbf{0}$ (a); Evolução das Cadeias de Markov para fluxo $\boldsymbol{q}(\boldsymbol{x})=\mathbf{5 0 0} \boldsymbol{W} / \boldsymbol{m}^{2}(\mathrm{~b})$; Histograma para $\boldsymbol{q}(\boldsymbol{x})=\mathbf{0}$ (c); e Histograma para

$$
\boldsymbol{q}(\boldsymbol{x})=500 \mathrm{~W} / \boldsymbol{m}^{2}(\mathrm{~d}) .
$$

Para a análise do erro na estimativa do fluxo de calor é utilizado o erro RMS, como definido a seguir nas Equações 19 e 20, para o fluxo de calor e o campo de temperaturas estimados. Os valores encontrados foram organizados na Tabela 1, correspondendo aos erros calculados para o fluxo de calor e as temperaturas.

$$
R M S_{q}=\sqrt{\frac{1}{N P_{x}} \sum_{i=1}^{N P_{x}}\left[q\left(x_{i}\right)-q_{i}\right]^{2}}
$$

e

$$
R M S_{T}=\frac{1}{N_{x}} \frac{1}{N_{t}} \sqrt{\sum_{i=1}^{N P_{x}} \sum_{k=1}^{N P_{t}}\left[T\left(x_{i}, t_{k}\right)-T_{i}^{k}\right]^{2}}
$$

Tabela 1. Erros calculados para o fluxo de calor e as temperaturas.

\begin{tabular}{c|c|c|c}
\hline Erro & Caso 1 & Caso 2 & Caso 3 \\
\hline$R M S_{q}$ & 323,518 & 84,2035 & 41,6954 \\
\hline$R M S_{T}$ & 0,0346 & 0,0313 & 0,0275 \\
\hline
\end{tabular}

Observando os valores dos erros RMS para cada caso fica claro como a modelagem das prioris na forma de TVD cumpre o papel de regularizar o problema de forma satisfatória. Apesar da utilização da técnica apenas como filtro não ter sido o suficiente para se conseguir estimar o fluxo, seu uso no Caso 2, sua aplicação nos candidatos do MCMC, conseguiu diminuir expressivamente o erro gerado. Mesmo não tendo gerado resultados satisfatórios, é possível notar que o uso do TVD nas medidas melhora significativamente os resultados da técnica aplicada aos candidatos, como é possível ver nos erros relativos 
ao Caso 3, que demonstram acurácia consideravelmente melhor do que aquela apresentada pelo Caso 2.

A Figura 4 apresenta o resíduo entre a temperatura exata e a estimada nos casos estudados, $\mathrm{em}^{\circ} \mathrm{C}$.

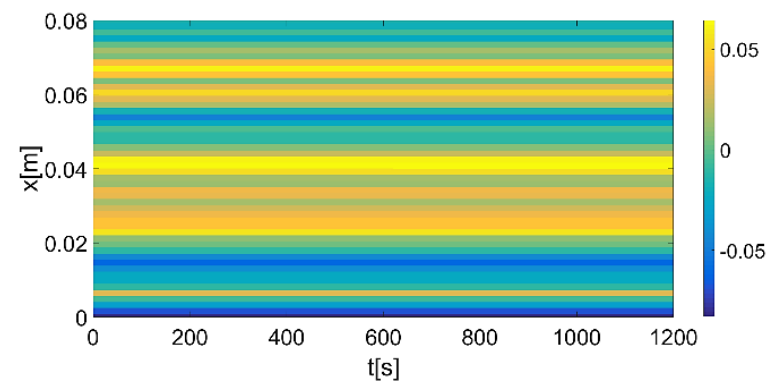

(a)

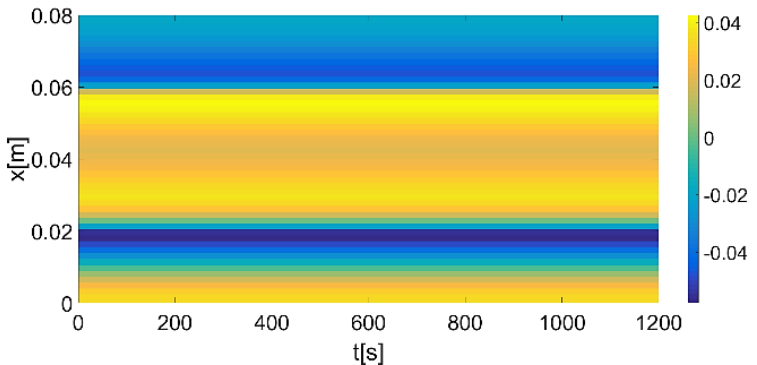

(b)

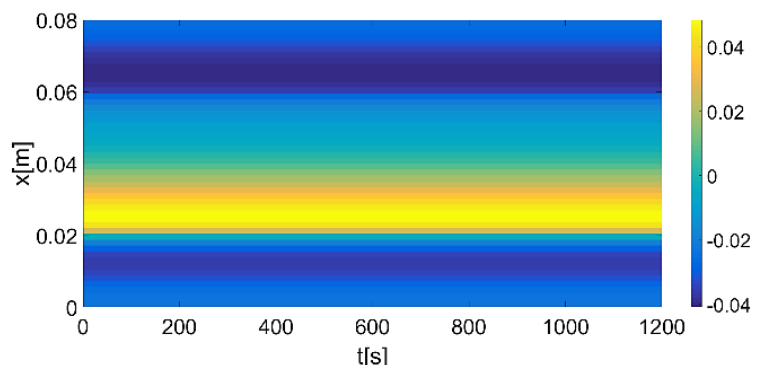

(c)

Figura 3. Resíduo entre temperaturas estimadas e medidas (em $\boldsymbol{C}^{o}$ ) para o Caso 1 (a), Caso 2 (b) e Caso 3 (c).

$\mathrm{Na}$ Figura 3(a) é possível notar um resíduo não correlacionado, enquanto que na Figura 3(b) percebe-se que o Caso 2 produziu um resíduo acentuado na zona onde há grande variação de fluxo e permaneceu abaixo do fluxo exato por quase toda a solução. Já no Caso 3, apresentado na Figura 3(c), é possível notar que, nas zonas onde a variação de fluxo é alta, resíduos de maior magnitude são produzidos, porém de forma mais branda do que no Caso 2. Esse resultado é compatível com o resultado para o fluxo de calor apresentado na Figura 2. Cabe ressaltar que no Caso 1, embora os resíduos sejam pequenos e pouco correlacionados, o fluxo estimado é muito contaminado pelo ruído experimental, conforme observa-se na Figura 2, resultado da amplificação dos ruídos experimentais (mesmo que tendo sido utilizado o TVD como filtro para estes dados) para a solução do problema inverso de estimativa do fluxo. 


\section{CONSIDERAÇÓES FINAIS}

Este trabalho tratou do problema inverso de estimativa do fluxo de calor unidimensional, utilizando o Método de Monte Carlo com Cadeias de Markov e regularização através de aplicações da técnica Total Variation Denoising. Os resultados da estimativa para o fluxo não se mostraram suficientemente regularizados com a utilização da técnica nas medidas. Por outro lado, a utilização da TVD, no segundo caso estudado, nos candidatos do MCMC apresentou resultados da estimativa de fluxo com boa concordância com o fluxo de calor imposto para a aplicação. A utilização das duas abordagens simultaneamente, no terceiro caso, conseguiu gerar resultados mais acurados do que no caso anterior, de acordo com o perfil de temperaturas e fluxo de calor estimados.

\section{AGRADECIMENTOS}

Os autores agradecem o suporte financeiro da FAPERJ, do CNPq e da CAPES. O segundo autor agradece o suporte financeiro do Instituto Federal Fluminense, instituição na qual é professor.

\section{REFERÉNCIAS}

ABREU, Luiz Alberto; ORLANDE, Hélcio; COLAÇO, Marcelo; Gaussian Hypermodel Applied to Failure Analysis of Laminated Composites. Proceedings of the 7th International Conference on Mechanics and Materials in Design. Portugal, 2017, p. $1183-1190$.

BERGMAN, Theodore; LAVINE, Adrienne; INCROPERA, Frank; DEWITT, David. Fundamentos de Transferência de Calor e de Massa. EUA: LTC Editora, 2014.

CONDAT, Laurent. A Direct Algorithm for 1D Total Variation Denoising. IEEE Signal Processing Letters. EUA, n. 20, 2013, p. 1054-1057.

KAIPIO, Jari; SOMERSALO, Erkki. Statistical and Computational Inverse Problems. EUA: Springer, 2005.

MESCOLIN, Marques; ABREU, Luiz Alberto; KNUPP, Diego; SILVA NETO, Antônio José. Estimation of Spatially and Time Varying Heat Flux via Markov chain Monte Carlo Method and Integral Transforms. Procedings of ENCIT (Encit 2016). Vitória, 2016

ORLANDE, Helcio; DULIKRAVICH, George; NEUMAYER, Markus; WATZENIG, Daniel; COLAÇO, Marcelo. Accelerated Bayesian Inference for the Estimation of Spatially Varying Heat Flux in a Heat Conduction Problem. Numerical Heat Transfer Part A-Applications. N. 1, vol. 65, 2014, p. 1-25. 
RUDIN, Leonid; OSHER, Stanley; FATEMI, Emad. Nonlinear Total Variation Based Noise Removal Algorithms. Physica D. N. 60, 1992, p. 259-268.

SILVA NETO, Antônio José; ÖZIŞIK, Necati. Simultaneous Estimation of Location and Timewise-Varying Strength of a Plane Heat Source. Numerical Heat Transfer Part AApplications. Inglaterra, n. 24, 2013, p. 467-477.

SU, Jian; SILVA NETO, Antônio José. Two-Dimensional Inverse Heat Conduction Problem of Source Strength Estimation in Cylindrical Rods. Applied Mathematical Modelling. Holanda, n. 25, 2001, p. 861-872. 